\title{
Rheological Behavior Analysis of Liver Fibrosis in Rats
}

\author{
Ying Zhu, Yuanyuan Shen, Xin Chen, Haoming Lin, and Siping Chen
}

\begin{abstract}
The process of liver fibrosis changes rheological properties of tissue. This study characterizes and compares two stages of liver fibrosis in rats. Two rheological models-Voigt model and Zener model are applied to the measured data. The experimental results demonstrate that Zener model is preferred to Voigt model for describing rheological properties of liver fibrosis stages $F 0$ and $F 2$ in rats.
\end{abstract}

Index Terms-Liver fibrosis, rheological properties, voigt model, zener model.

\section{INTRODUCTION}

Liver fibrosis is the widespread disease worldwide. The liver have been invaded by various pathogen, causing liver damage and inflammation, at the same time the immune system of liver tissue is activated. Liver fibrosis is a repair process of the damaged tissue, which refers to the accumulation of extracellular matrix(ECM) proteins. Currently liver biospy is still the gold standard for the diagnosis of liver fibrosis. Fibrosis grading has been evaluated semiquantitatively according to the METAVIR scoring system: F0, no fibrosis; F1, portal fibrosis without septae; F2, portal fibrosis and few septae; F3, numerous septae without cirrhosis; F4, cirrhosis [1]. The alterations of tissue pathological status means its biomechanics properties are changed. Feng [2] regarded viscoelasticity as the best indicator of soft tissue mechanics properties. As everyone knows, obtaining viscoelaticity parameters quantificationally depends on the appropriate rheological model of describing soft tissue. Generally, Voigt model is often used to describe rheological properties of normal soft tissue [3]-[9], which has one elasticity parameter and one viscocity parameter. However, due to pathological changes, does Voigt model explain appropriately rheological behavior of liver fibrosis at different stages? So far, there are no studies reported in liteature on rheological properties of liver fibrosis. This study performed rheological mechanical experiments to confirm appropriate rheological model for fibrosis stages F0 and F2 in rats.

\section{THEORY AND EXPERIMENTS}

Rheology experiments describe the dynamic mechanical

Manuscript received November 14, 2012; revised February 16, 2013 This work was supported by the National Natural Science Foundation of China under Grant 61031003.

Ying Zhu is with National-Regional Key Technology Engineering Laboratory for Medical Ultrasound, Shenzhen University, Shenzhen, Guangdong, 518060, China (e-mail: jgwenzying@yahoo.com.cn).

Yuanyuan Shen and Siping Chen are with Department of Biomedical Engineering, School of Medicine, Shenzhen University, Shenzhen, Guangdong, 518060, China (e-mail: shenyy@szu.edu.cn, chensiping@szu.edu.cn). behavior of biological tissue. A sinusoidal shear strain $\varepsilon(t)=\varepsilon_{0} e^{i \omega t}$ is imposed on the tissue, then a sinusoidal shear stress $\sigma(t)=\sigma_{0} e^{i(\omega t+\delta)}$ is induced at the same frequency. The ratio of sinusoidal strain and sinusoidal stress is represented by the complex shear modulus $G^{*}(\omega)$

$$
\begin{aligned}
G^{*}(\omega) & =\frac{\sigma_{0} e^{i(\omega t+\delta)}}{\varepsilon_{0}(t) e^{i \omega t}}=\frac{\sigma_{0}}{\varepsilon_{0}} e^{i \delta} \\
= & \frac{\sigma_{0}}{\varepsilon_{0}}(\cos \delta+i \sin \delta)=G_{1}(\omega)+i G_{2}(\omega) .
\end{aligned}
$$

where $\varepsilon_{0}$ is shear strain amplitude, $\sigma_{0}$ is shear stress amplitude, $\omega_{0}$ is angular frequency, $\delta$ is a phased-shifted angle. $G_{1}(\omega)$ is the storage modulus, $G_{2}(\omega)$ is the loss modulus, the magnitude of $G^{*}(\omega)$ is

$$
|G(\omega)|=\sqrt{G_{1}^{2}(\omega)+G_{2}^{2}(\omega)} .
$$

Voigt model and Zener model are used in this study, as shown in Fig. 1 (a) and Fig. 1 (b). Voigt model is a common rheological model of soft tissue, which consists of an elastic spring $E_{1}$ and a viscous damper $\eta$ connected in parallel. Zener model consists of an elastic spring $E_{2}$ and a viscous damper $\eta$ connected in series, then with spring $E_{1}$ in parallel. Their complex shear moduli are respectively expressed as

$$
\begin{gathered}
G_{V}^{*}(\omega)=E_{1}+i \omega \eta . \\
G_{Z}^{*}(\omega)=\left(E_{1}+\frac{\omega^{2} \eta^{2} E_{2}}{E_{2}^{2}+\omega^{2} \eta^{2}}\right)+i \frac{\omega \eta E_{2}^{2}}{E_{2}^{2}+\omega^{2} \eta^{2}}
\end{gathered}
$$

Thus the magnitude of the complex shear modulus can be expressed as

$$
\begin{gathered}
\left|G_{V}^{*}(\omega)\right|=\sqrt{E_{1}^{2}+\omega^{2} \eta^{2}} \\
\left|G_{Z}^{*}(\omega)\right|=\sqrt{E_{1}^{2}+\frac{\left(2 E_{1}+E_{2}\right) E_{2} \omega^{2} \eta^{2}}{E_{2}^{2}+\omega^{2} \eta^{2}}} .
\end{gathered}
$$

In this experiments, 16 Male Sprague-Dawley rats (provided by Guangdong Medical Laboratory Animal Center, Foshan, Guangdong) weighing 180-270g were used. These rats were randomized into 2 groups with 6 members in the control group and 10 members in the model group inducing liver fibrosis. About $50 \%$ carbon tetrachloride $\left(\mathrm{CCl}_{4}\right)$ in olive oil was injected subcutaneously in 10 rats twice a week with $0.3 \mathrm{ml} / 100 \mathrm{~g}$ weight. The concentration was doubled at the 
first time and adjusted according to the rat weight for the rest of time. After the 5th-8th weeks, rats in liver fibrosis stage F2 were obtained. Fibrosis grading for each rat was identified by pathological section and trichrome masson staining. The modeling of liver fibrosis stage F2 were successful in 8 members of 10 rats, the rest were for stage F1. All the procedures of studies were approved by Animal Care committee of Shenzhen University and Guangdong Medical Laboratory Animal Center.

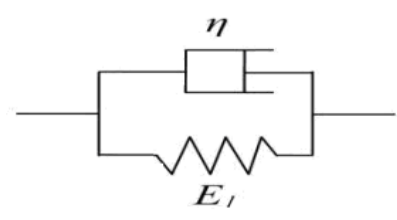

(a)

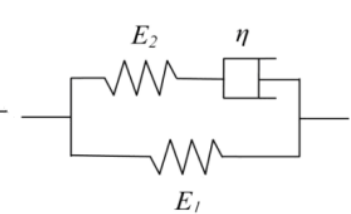

(b)
Fig. 1. (a) Voigt model. (b) Zener model.

To quantify the dynamic mechanical behavior of the rats' liver, rheometer tests were performed in sequence of fibrosis stages F0 and F2. The tests in small deformations (linear domain) were carried out at room temperature $\left(23 \pm 1^{\circ} \mathrm{C}\right)$ using a strain-controlled rheometer (AR1000, TA Instruments, New Castle, DE,USA) using its $25 \mathrm{~mm}$ - diameter parallel plates configuration. The livers were harvested after euthanasia for the rats. Each rat liver was extracted to 1 or 2 specimens, thus total liver samples were 16 pieces, including 6 pieces for $\mathrm{F} 0,10$ pieces for $\mathrm{F} 2$. The specimens, which were $4 \pm 1 \mathrm{~mm}$ thickness, were placed between the plates and the edges were carefully trimmed with a scalpel. First, the tissue linear behavior domain was determined by performing strain sweep oscillation tests at $1 \mathrm{~Hz}$ and $40 \mathrm{~Hz}$ respectively with the strain amplitude increasing from $0.01 \%$ to $2 \%$. Then frequency sweep oscillations were carried out at the fixed strain $0.5 \%$ from $1 \mathrm{~Hz}$ to $40 \mathrm{~Hz}$. Finally, the magnitude of complex moduli $\left|G^{*}(\omega)\right|$ values was obtained.

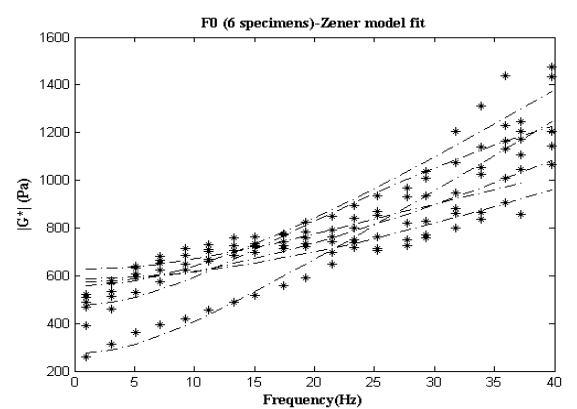

(a)

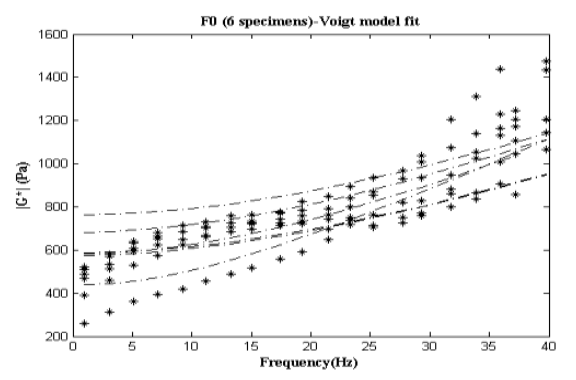

\section{RESUlTS AND DISCUSSION}

Fig. 2 (a) shows $\left|G^{*}(\omega)\right|$ values for rat livers in fibrosis stages F0 and F2, represented by symbol “*”, meanwhile, the $\left|G^{*}(\omega)\right|$ values are fitted to Zener model using LevenbergMarquardt method for nonlinear least squares fitting and are represented by the dashed line. The $\left|G^{*}(\omega)\right|$ data are also fitted to the conventional Voigt model by the dashed line, shown in Fig. 2 (b). Viscoelasticity parameters of the models and the coefficient of determination $R^{2}$ are given in table I . $R^{2}$ is ratio of regression deviation and total deviation which is regarded as evaluation indicator for goodness of fit.

Considering vertical coordinate in Fig. 2 (a) and (b), $\left|G^{*}(\omega)\right|$ is ranged from $200 \mathrm{~Pa}$ to $500 \mathrm{~Pa}$, which indicated liver rheological properties are changed with fibrosis stages. According to $R^{2}$ in Table $\mathrm{I}$, it is obvious that Zener model provides better regression than Voigt model for stage F2, though the two models are equal regression for stage F0. Equation (6) is approximately equal to (5) due to $E_{2}>E_{1}$ in stage $\mathrm{F} 0$, thus the two models can both explain the rheological behavior of rats liver in stages F0.However, in stage F2, the order of magnitudes of $E_{2}$ is close to $E_{1}$, so (6) is not approximately equal to (5) any more, which depended on the relationship of $E_{1}$ and $E_{2}$. The change of ratio $E_{2} / E_{1}$ reveals that liver elasticity changed from stages F0 to F2.Voigt model is not appropriate for describing rheological properties for stage F2. But better agreement between fit and experiments is achieved by using Zener model for stages F0 and F2. In conclusion, the experimental results demonstrate that Zener model can describe tissue rheological behavior of rats' liver in fibrosis stages F0 and F2 successfully.

(b)
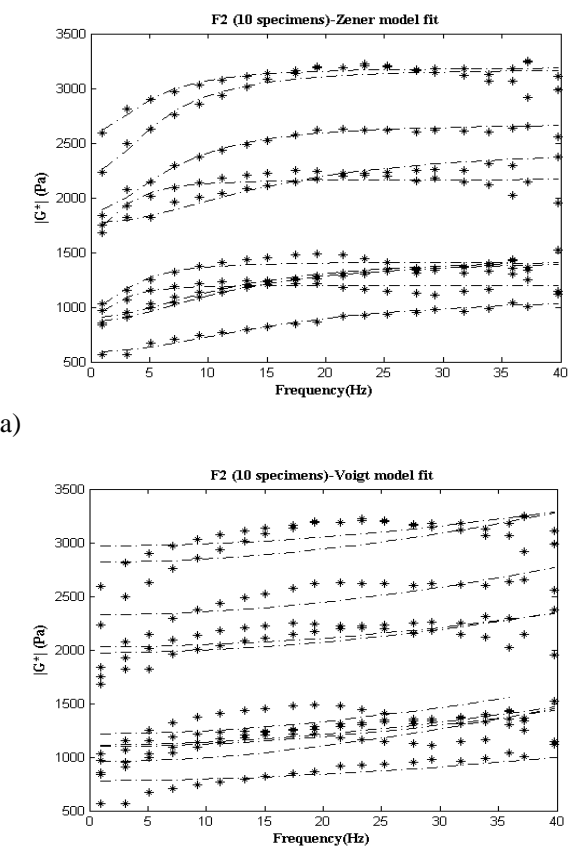

Fig. 2. The experiments results of stages F0 and F2, fitted to (a)Zener model (b) Voigt model. 
TABLE I: THE VISCOELASTICITY PARAMETERS ACCORDING TO THE MODELS

\begin{tabular}{|c|c|c|c|c|c|c|c|c|}
\hline \multirow[b]{2}{*}{ Stage } & \multirow{2}{*}{ Specimen } & \multicolumn{4}{|c|}{ Zener model } & \multicolumn{3}{|c|}{ Voigt model } \\
\hline & & $E_{1}(\mathrm{~Pa})$ & $E_{2}(\mathrm{~Pa})$ & $\eta(\mathrm{Pa} \cdot \mathrm{s})$ & $R^{2}$ & $E_{1}(\mathrm{~Pa})$ & $\eta(\mathrm{Pa} \cdot \mathrm{s})$ & $R^{2}$ \\
\hline \multirow{6}{*}{ F0 } & 1 & 272.245 & $7.89 \mathrm{E}+06$ & 4.858 & 0.9453 & 272.244 & 4.859 & 0.9453 \\
\hline & 2 & 626.201 & 982.169 & 2.532 & 0.9160 & 638.537 & 3.332 & 0.9160 \\
\hline & 3 & 558.042 & $9.14 \mathrm{E}+06$ & 5.019 & 0.9199 & 558.042 & 5.020 & 0.9199 \\
\hline & 4 & 474.575 & 1378.9 & 4.495 & 0.9773 & 517.875 & 4.742 & 0.9633 \\
\hline & 5 & 573.790 & $7.35 \mathrm{E}+06$ & 3.673 & 0.8530 & 573.790 & 3.674 & 0.8530 \\
\hline & 6 & 587.495 & $3.47 \mathrm{E}+05$ & 3.029 & 0.9022 & 587.215 & 3.036 & 0.9022 \\
\hline \multirow{10}{*}{$\mathrm{F} 2$} & 1 & 2591.6 & 602.399 & 18.319 & 0.9483 & 2984.5 & 5.386 & 0.3266 \\
\hline & 2 & 590.952 & 524.008 & 4.228 & 0.9612 & 690.776 & 3.573 & 0.8503 \\
\hline & 3 & 1692.4 & 477.381 & 24.523 & 0.9105 & 2114.3 & 0.898 & 0.1263 \\
\hline & 4 & 1771.2 & 693.606 & 6.484 & 0.8599 & 1926.1 & 6.484 & 0.7566 \\
\hline & 5 & 1872.4 & 813.114 & 16.740 & 0.9158 & 2294.2 & 6.607 & 0.4912 \\
\hline & 6 & 2233 & 952.026 & 23.384 & 0.9294 & 2813.9 & 6.852 & 0.3154 \\
\hline & 7 & 985.000 & 430.156 & 18.115 & 0.9552 & 1328.1 & 2.029 & 0.2786 \\
\hline & 8 & 923.198 & 279.004 & 17.233 & 0.9277 & 1151.8 & 1.652 & 0.2701 \\
\hline & 9 & 907.822 & 541.779 & 6.572 & 0.9526 & 1080.7 & 4.335 & 0.7497 \\
\hline & 10 & 871.412 & 563.783 & 6.612 & 0.9243 & 1047.6 & 4.360 & 0.7139 \\
\hline
\end{tabular}

\section{ACKNOWLEDGMENT}

Ying Zhu thanks Dayong Gui, Xue Gao from the College of Chemistry and Chemical Engineering for the rheometer.

\section{REFERENCES}

[1] French METAVIR Cooperative Study Group, "Intraobserver and interobserver variations in liver biopsy interpretation in patients with chronic hepatitis C," Hepatology, vol. 20, no. 1 Pt 1, pp. 15-20,1994.

[2] Y.C. Fung, Biomechanics: mechanical properties of living tissues, 2nd ed., Springer, 1993, ch. 2, pp. 47-61.

[3] D. Valtorta and E. Mazza, "Measurement of rheological properties of soft biological tissue with a novel torsional resonator device," Rheol. Acta, vol. 45, no. 5, pp. 677-692, 2006.

[4] Z. Liu and L. Bilston, "On the viscoelastic character of liver tissue: experiments and modelling of the linear behaviour," Biorheology, vol. 37, no. 3, pp. 191-201, 2000.

[5] S. Nicolle, M. Lounis, R. Willinger, and J. F. Palierne, "Shear linear behavior of brain tissue over a large frequency range," Biorheology, vol. 42, no. 3, pp. 209-223, 2005.

[6] S. Chatelin, J. Oudry, N. Périchon, L. Sandrin, P. Allemann, L. Soler, and R. Willinger, "In vivo liver tissue mechanical properties by Transient Elastography: comparison with dynamic mechanical analysis," Biorheology, vol. 48, no. 2, pp. 75-88, 2011.

[7] D. Klatt, C. Friedrich, Y. Korth, R. Vogt, J. Braun, and I. Sack, "Viscoelastic properties of liver measured by oscillatory rheometry and multifrequency magnetic resonance elastography," Biorheology, vol 47, no. 2, pp. 133-141, 2010.

[8] S. G. Chen, M. Fatemi, and J. F. Greenleaf, "Quantifying elasticity and viscosity from measurement of shear wave speed dispersion," J. Acoust. Soc. Am., vol. 115, no. 6, pp. 2781-2785, 2004.
[9] H. L. Oestreicher, "Field and impedance of an oscillating sphere in a viscoelastic medium with an application to biophysics," J. Acoust. Soc. Am., vol. 23, no. 6, pp. 707-714, 1951.

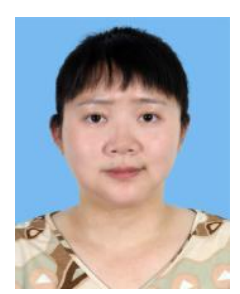

Ying Zhu was born in Jan. 1980. She is currently a Ph.D. student in National-Regional Key Technology Engineering Laboratory for Medical Ultrasound, Shenzhen University, Guangdong, China. Her research interests include biomechanics and ultrasound elasticity imaging.

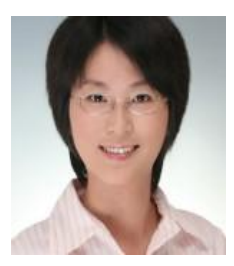

Yuanyuan Shen was born in Feb. 1983. She received B. Eng. degree and Ph.D degree in biomedical engineering from Shanghai Jiao Tong University, Shanghai, China in 2003 and 2009. Her current research interests include ultrasound elastography and microbubble mediated drug delivery.

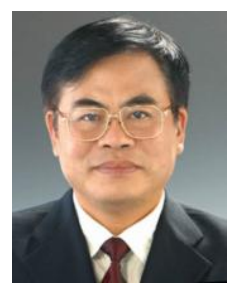

Siping Chen was born in Dec. 1948. He received Ph.D. degree in biomedical engineering from Xi'an Jiao Tong University,Xi'an,P.R.China in 1987. He is the $\mathrm{Ph} . \mathrm{D}$. supervisor in biomedical engineering and the chief of National-Regional Key Technology Engineering Laboratory for Medical Ultrasound of Shenzhen University. His research interests include untrasound imaging, ultrasonic engineering and biomedical signal processing. 